\title{
A Simplified Technique for Fabrication of One Piece Closed Hollow Bulb Obturator for Rehabilitating Young Adult with Total Maxillectomy: A Clinical Report
}

\author{
Dhital $\mathrm{S}^{1^{*}}$, Mathema $\mathrm{S}^{2}$ \\ ${ }^{1}$ Resident, Department of Prosthodontics, People's Dental College and Hospital, Kathmandu, Nepal. \\ ${ }^{2}$ Professor, Department of Prosthodontics, People's Dental College and Hospital, Kathmandu, Nepal.
}

\begin{abstract}
Rehabilitation of patients with a palatal defect requires management of anatomical, functional, esthetics as well as psychological factors. A reduced denture-bearing area, increased inter arch space, and cantilever movement cause complex problems in these palatal defects. Solid obturator is heavy and continuous stresses on the remaining tissues jeopardizes the health, compromise the function of the prosthesis and affect patient comfort. Reducing the weight of the prosthesis by making it hollow prevents the cantilever movement thereby increasing retention, stability and brings improvement in speech by resonance effect. This clinical report describes the rehabilitation of young adult patient with total maxillectomy using maxillary hollow bulb obturator fabricated with lost salt technique.
\end{abstract}

Key words: Hollow bulb obturator, Maxillectomy.

\section{Introduction}

$\mathrm{R}$ ehabilitation of total maxillectomy patient is a challenging task. Overcoming anatomical and functional limitations along with psychological management is the prime concern during rehabilitation of these types of defects. The surgery often includes part of hard as well as soft palate removal and results in an oro-antral and or oro-nasal communication. ${ }^{1}$ Maxillary obturators is more common treatment options for rehabilitating these defects when surgical closure is not possible or due to the failure of the surgery. ${ }^{2}$ Increased weight of the maxillary obturator often cause the prosthesis to act as a cantilever. ${ }^{3}$ Hence ,obturator should be light in weight to provide retention, stability, support, comfort and to maintain hygiene. A hollow maxillary obturator may reduce the weight of

\section{*Corresponding Author}

Dr. Shaishab Dhital, Resident, Department of Prosthodontics and Maxillofacial Prosthetics, People's Dental College and Hospital, Kathmandu, Nepal.

E-mail: shaishavdhital@gmail.com the prosthesis by up to $33 \%$, depending upon the size of the maxillary defect as stated by Minsley et al. ${ }^{4}$

Many methods had been employed for fabricating a hollow bulb obturator. The classic method is to fabricate the prosthesis and subsuently grinding out the unwanted portion and cover it. ${ }^{5}$ Chalian and Barnett suggested making of a hollow shim. ${ }^{6}$ Palmer and Coffey used clay to simulate the ridge contour for fabrication of hollow bulb obturator. ${ }^{7}$ Others have suggested a two flask investing technique with an autopolymerizing seal between the two halves. All of these methods are long and tedious and require the assistance of a knowledgeable dental laboratory technician. ${ }^{6}$ In this case report a patient who had undergone total maxillectomy was rehabilitated with a hollow bulb obturator using a lost salt technique which is simple and easy to execute.

\section{Clinical Report}

A 15-year-old male, was referred to the Department of Prosthodontics and Maxillofacial 
Prosthetics, People's Dental College and Hospital, Sorakhutte, Kathmandu for the fabrication of prosthesis to correct an acquired palatal defect. The patient had undergone surgery 18 months back for the removal of a juvenile nasopharyngeal angiofibroma involving a portion of the hard palate followed by radiotherapy (Fig.1). A complete review of the patient's history and oral examination was done. The intra-oral examination revealed a left total maxillectomy extending from the right central incisor to the left second molar which indicated Aramany class I defect. ${ }^{8}$ The different treatment options were discussed with the patient for the rehabilitation of the palatal defect. Due to growing age of the patient, fabrication of a long term interim closed hollow bulb obturator was planned.

A stock metal perforated impression tray (Impression Trays, Samit, New Delhi, India) of proper size was selected. Modification was done by using impression compound (Impression Composition, Samit, New Delhi, India) with occlusal stops anteriorly and posteriorly in dentate side for the purpose of reorientation of the tray and support for impression material (Fig.2). Impression of maxillary and mandibular arches were made with alginate (Zelgan 2002, Dentsply India, Haryana, India) (Fig.3) and poured with Type III dental stone (Kalstone, Kalabhai Karson Pvt. Ltd, Mumbai, India). The undercut of the defect over the cast was blocked by modelling wax (Modelling Wax, Pyrex, India) (Fig.4). Fitted labial bow was fabricated with 20 guage stainless steel wire (Samit Products, New Delhi, India) (Fig.4). 2 mm thick layer of autopolymerizing acrylic resin (Rapid Repair, Pyrex, India) was lined over the defect with hollow space in the center (Fig.5). The center space was then filled with table salt to fill the concavity (Fig.6). Lid over the defect was fabricated with autopolymerizing acrylic resin extending from the margins covering over the salt.
Holes were made in the superior surface of the acrylic resin using a bur. Table salt inside the space was removed by flushing water under high pressure by syringe. (Fig.7). These access holes were then sealed with autopolymerizing acrylic resin. Occlusal wax rim was fabricated over this hollow bulb obturator using modelling wax (Fig.8). Maxillo-mandibular relationship, articulation, teeth arrangement (Fig.9), try-in, and processing, finishing and polishing were done by conventional manner. The hollow bulb obturator was thus fabricated with inner core of self- cure acrylic resin and surface processed with heat cure resin (Trevalon, Dentsply India, India) (Fig.10, 11 and 12).

The obturator was then inserted intra-orally to evaluate the fit, verification of the occlusion and esthetic. (Fig.13). The patient was trained to do insertion and removable of the prosthesis and instructed to masticate on the non-resected side only. Maintaining hygiene, limitation of the prosthesis and recall protocol was explained. Recall and follow up was done after 1 week, 1 month, 3 months and 6 months. Functional, esthetic and psychological comfort of the patient could clearly be perceived during his follow-up visits.

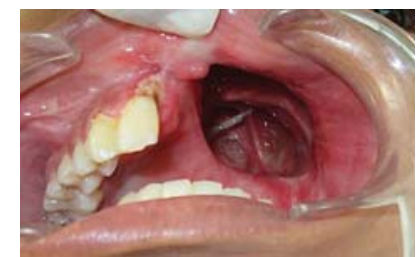

Figure 1: Intraoral occlusal view

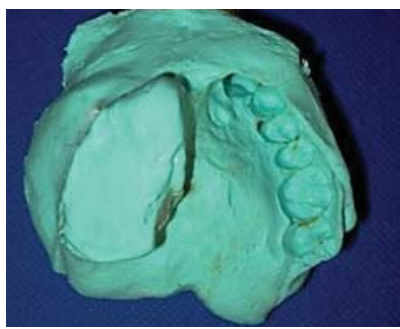

Figure 3: Alginate impression

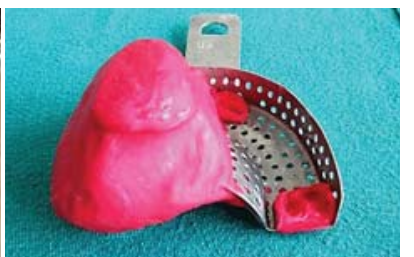

Figure 2: Tray modification with impression compound

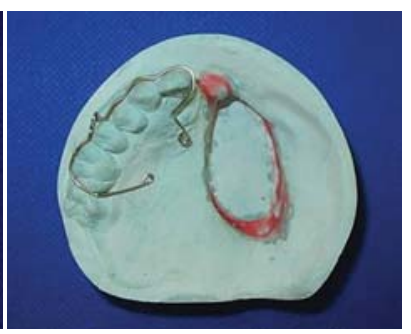

Figure 4: Cast with fitted labial bow 


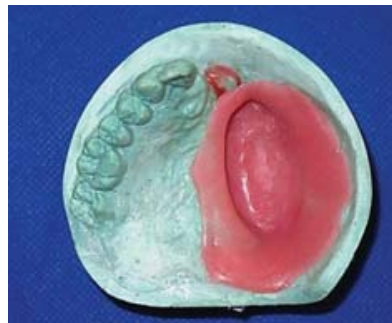

Figure 5: The defect area was lined with approximately 2

mm thick layer of autopolymerizing acrylic resin

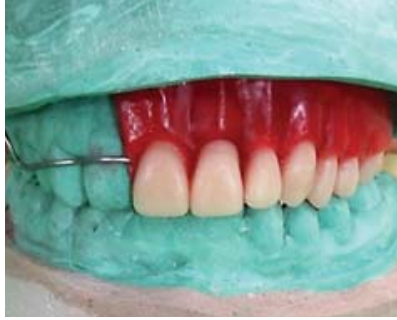

Figure 9: Teeth arrangement

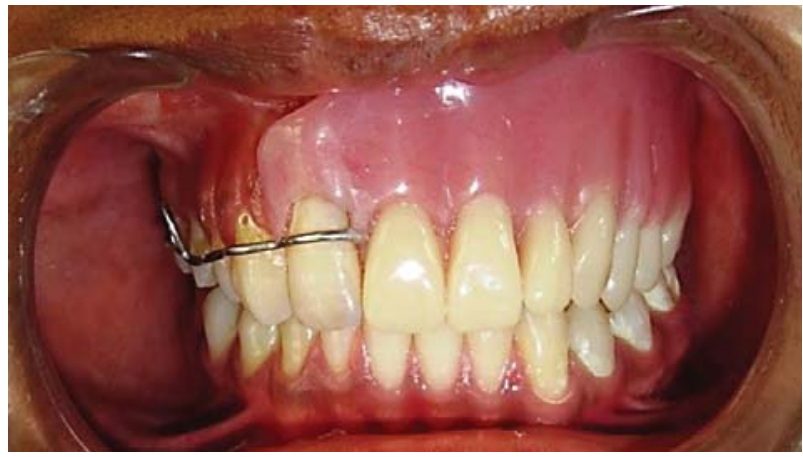

Figure 13: Closed hollow bulb obturator delivery

\section{Discussion}

A wide variety of treatment options are available for prosthetic rehabilitation of total maxillectomy cases which includes surgical reconstruction, surgical reconstruction followed by prosthetic rehabilitation and a nonsurgical approach involving only prosthetic rehabilitation. A closed hollow bulb obturator was fabricated in this patient since primary closure of the defect with surgical reconstruction was not feasible. Further literature does not suggest improvement in the overall treatment

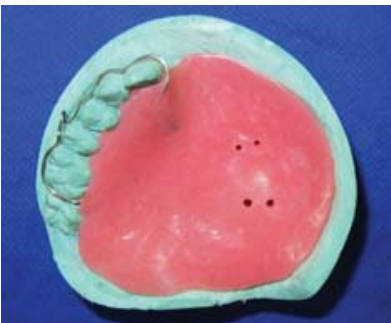

Figure 7: Holes made on the superior surface of the obturator acrylic resin

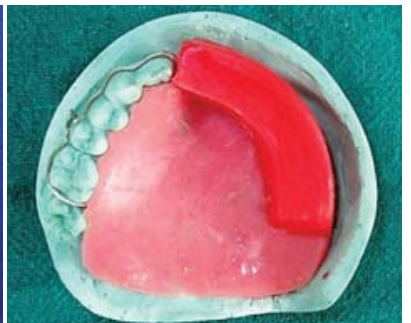

Figure 8: Fabrication of occlusal wax rim

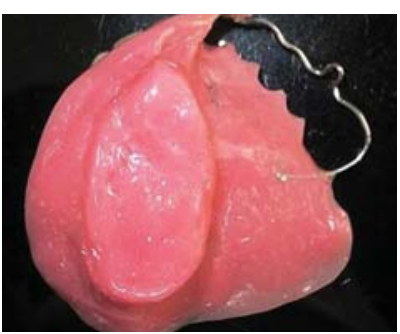

Figure 11: Intaglio surface

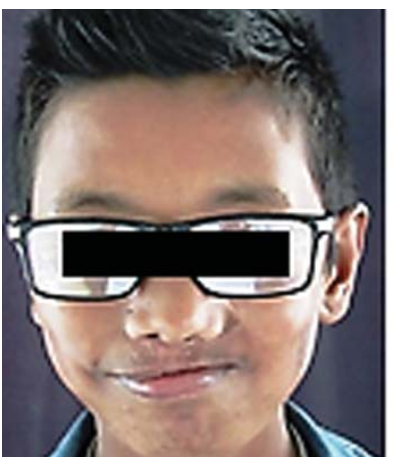

Figure 14: Extraoral frontal view, pre and postdelivery of closed hollow bulb obturator

outcome. ${ }^{2}$ Alternatively, open type of hollow obturator was not fabricated since a closed hollow bulb obturator was more hygienic, enabled easy maintenance with reduced weight of the prosthesis and more importantly, maximum coverage of the defect which further aided in retention, support and stability., 3,10 Lost salt technique has been utilised to achieve the hollow part of the obturator since it is less technique sensitive, easy and economical with assurance of approximate wall thickness. A dental implant-supported prosthesis and 
definitive prosthesis were not considered due to the growing age of patient.

\section{Summary}

This clinical report described a novel and simple procedure for fabrication of closed hollow bulb obturator for prosthetic rehabilitation of a patient with a total maxillectomy. The decreased weight of the prosthesis provides retention, stability and improves speech by resonance effect, thus enhancing both form and function.

\section{References}

1. Ortegon SM, Martin JW, Lewin JS. A hollow delayed surgical obturator for a bilateral subtotal maxillectomy patient: a clinical report. The Journal of prosthetic dentistry. 2008;99(1):1418.

2. Pigno MA. Conventional prosthetic rehabilitation after free flap reconstruction of a maxillectomy defect: a clinical report. The Journal of prosthetic dentistry. 2001;86(6):578581.

3. El Mahdy A. Processing a hollow obturator. The Journal of prosthetic dentistry. 1969;22(6):682686.
4. Minsley GE, Nelson DR, Rothenberger SL. An alternative method for fabrication of a closed hollow obturator. The Journal of prosthetic dentistry. 1986;55(4):485-490.

5. Habib BH, Driscoll CF. Fabrication of a closed hollow obturator. The Journal of prosthetic dentistry. 2004;91(4):383-385.

6. Chalian V, Barnett M. A new technique for constructing a one-piece hollow obturator after partial maxillectomy. The Journal of Prosthetic Dentistry. 1972;28(4):448-453.

7. Palmer B, Coffey KW. Fabrication of the hollow bulb obturator. The Journal of prosthetic dentistry. 1985;53(4):595-596.

8. Aramany MA. Basic principles of obturator design for partially edentulous patients. Part I: Classification. Journal of Prosthetic Dentistry. 2001;86(6):559-561.

9. Keyf F. Obturator prostheses for hemimaxillectomy patients. Journal of oral rehabilitation. 2001;28(9):821-829.

10. Wu Y-1, Schaaf NG. Comparison of weight reduction in different designs of solid and hollow obturator prostheses. The Journal of prosthetic dentistry. 1989;62(2):214-217. 